\title{
La acción concertada de la Comunidad Internacional para la protección del patrimonio cultural en conflictos armados: Mali como paradigma
}

\author{
The concerted action of the international community for the protection \\ of cultural heritage in armed conflicts: Mali as a paradigm
}

RECIBIDO: 23 DE SEPTIEMBRE DE 2016 / ACEPTADO: 3 DE OCTUBRE DE 2016

\section{Félix VACAS FERNÁNDEZ}

Profesor titular de Derecho Internacional Público y Relaciones Internacionales

Universidad Carlos III de Madrid

fvacas@der-pu.uczm.es

Resumen: En Mali, por primera vez, se ha producido la actuación concertada y decidida de la Comunidad Internacional para la protección del patrimonio cultural en un país en situación de conflicto armado: el Consejo de Seguridad, UNESCO y la Corte Penal Internacional han mostrando su capacidad de responder a los graves desafíos que en esta materia afronta la Humanidad y han demostrado que con voluntad política y actuación conjunta y coordinada se puede proteger, reconstruir y conservar eficazmente el patrimonio de un pueblo; que es proteger, reconstruir y conservar la identidad del pueblo mismo y, en definitiva, la paz de las personas que lo conforman.

Palabras clave: Patrimonio cultural y conflictos armados, UNESCO, MINUSMA, Corte Penal Internacional.

\begin{abstract}
In Mali, for the first time ever, there has been a concerted and determined action of the International Community in order to protect the cultural heritage of a country under armed conflict: the Security Council, UNESCO and the International Criminal Court have shown their ability to answer the serious challenges that Humanity faces in this field; and have proved that, with political will and through common and coordinated action, the heritage of a people can be effectively protected, rebuilt and preserved, which amounts to protect, rebuild and preserve the identity of the people and, ultimately, peace itself
\end{abstract}

Keywords: Cultural heritage and armed conflict, UNESCO, MINUSMA, International Criminal Court.

Sumario: CONSIDERACIONES PREVIAS. 1. LAACTUACIÓN DE LAS NACIONES UNIDAS: LA MISIÓN DE LAS NACIONES UNIDAS EN MALI (MINUSMA), PRIMERA OPERACIÓN DE PAZ EN CUYO MANDATO SE INCLUYE LA PROTECCIÓN DEL PATRIMONIO CULTURAL. 2. LA ACTUACIÓN DE LA UNESCO EN MALI. 2.1. La labor de concienciación y capacitación del personal de la MINUSMA para la protección del patrimonio cultural de Mali. 2.2. La acción de la UNESCO para la reconstrucción del patrimonio cultural de Mali destruido o dañado en el conflicto. 3. LA ACTUACIÓN DE LA CORTE PENAL INTERNACIONAL: LA LUCHA CONTRA LA IMPUNIDAD EN MATERIA DE PROTECCIÓN DEL PATRIONIO CULTURAL. 3.1. La situación de Mali ante la Corte Penal Internacional. 3.2. El asunto Fiscalía v. Ahmad Al Faqi Al Mahdi: primer caso ante la Corte Penal Internacional de crímenes de guerra por la destrucción de bienes protegidos. CONSIDERACIONES FINALES. 


\section{CONSIDERACIONES PREVIAS}

E 1 abrupto final de la primera fase de la guerra en Libia, propiciado por la intervención militar occidental liderada por Francia y el Reino Unido -e irónicamente legitimada bajo el, aparentemente positivo, por humano, principio de responsabilidad de proteger $^{1}$ sobre el que se fundamenta expresamente la resolución 1973 (2011), de 17 de marzo, del Consejo de Seguridad que autorizó en ese país el uso de la fuerza coercitiva ${ }^{2}-$, ha traído no pocas consecuencias negativas a la cuenca del Mediterráneo, al Sahara y al Sahel. Entre ellas, la guerra en Mali.

1 Entre la muy abundante bibliografía sobre la responsabilidad de proteger, vid. BANNON, A. L., «The Responsibility to Protect: The U.N. World Summit and the question of unilateralism», 115 Yale Lane Fournal, 1157-1164 (2006); Bellamy, A., Global politics and the responsibility to protect. From words to deeds, Routledge, 2011; BERMEJO GARCÍA, R., «De la intervención humanitaria a la responsabilidad de proteger: fundamentos, similitudes y diferencias», en C. GUTIÉRREZ EsPaDa (dir.), Darfur, el conflicto inacabable, Thomson Reuters Aranzadi, The Global Law Collection, Navarra, 2012; BRITO, W. L., «A responsabilidade de proteger da comunidade internacional: pressupostos e fins», en Estudios de Derecho Internacional y de Derecho Europeo en Homenaje al Profesor Manuel Pérez González, Tirant lo Blanch, Valencia, 2012; CoOPER R. H. y Kohler, J. V. (eds.), Responsibility to Protect: The Global Moral Compact for the 21st Century, Palgrave Macmillian, London, 2009; Evans, G., The responsibility to Protect: Ending Mass Atrocity Crimes. Once and for all, Brookings Institution Press, Washington DC, 2008; GARCÍA PÉREZ, R., «La 'responsabilidad de proteger': un nuevo papel para Naciones Unidas en la gestión de la seguridad internacional», Revista Electrónica de Estudios Internacionales (2006), pp. 1 ss.; GUTIÉRREZ ESPADA, C., «Sobre la responsabilidad de proteger (RP) y el uso legal de la fuerza», en F. Vacas Fernández (dir.), Comunidad Internacional, Oriente Próximo y Cultura de Paz, no 3 Cuadernos Cultura de Paz, Instituto de Estudios Internacionales y Europeos «Francisco de Vitoria» de la Universidad Carlos III de Madrid, 2015, pp. 7-18; LEVITT, J. I., «The Responsibility, to protect: A Beaver without a dam?», 25 Michigan fournal of International Law 153-177 (2003); LÓPEZ-JACOISTE DíAZ, E., «La responsabilidad de proteger: Reflexiones sobre su fundamento y articulación», 22 Anuario Español de Derecho Internacional 285 (2006); MARCLAY, E., La Responsabilité de Protéger: Un nouveau paradigme ou une boîte à outils?, Étude Raoul-Dandourand $\mathrm{n}^{\mathrm{o}} 10$, 2005; SÁNCHEZ PATRÓN, J. M., «La responsabilidad de proteger: reflexiones críticas en torno a cuestiones clave», 167 Estudios Internacionales 75 (2010).

2 Sobre la aplicación de la responsabilidad de proteger en Libia vid. BERMEjo García, R., «La protección de la población civil en Libia como coartada para derrocar un gobierno: un mal inicio para la responsabilidad de proteger», 27 Anuario Español de Derecho Internacional 9-55 (2011); GutiÉRreZ EsPADA, C., «Sobre el 'núcleo duro' de la Resolución 1973 (2011) del Consejo de Seguridad y acerca de su aplicación práctica», 27 Anuario Español de Derecho Internacional (2011); LóPEZ-JaCOISTE DíAz, E., «La crisis de Libia desde la perspectiva de la responsabilidad de proteger», 27 Anuario Español de Derecho Internacional (2011); Oliva MarTínEZ, D., «Cuestiones en torno a la legitimidad del Consejo de Transición Libio a raíz de su reconocimiento por la Asamblea General de Naciones Unidas», 27 Anuario Español de Derecho Internacional 153-183 (2011). 
En efecto, Mali -Estado surgido del proceso de descolonización de la mano del principio uti possidetis iuris ${ }^{3}$ en 1960, con grandes carencias en su (falta de) desarrollo económico y social, así como con una profunda desestructuración de origen por la falta de cohesión entre las distintas regiones que lo conforman y, sobre todo, entre los diferentes pueblos que lo habitan ${ }^{4}-$ ha venido experimentando desde entonces sucesivas rebeliones tuaregs, en el Norte, que siempre han pretendido una mayor autonomía de Bamako, cuando no la independencia 5 . Sin embargo, la rebelión que se inició en enero de 2012 superará en gravedad a las anteriores tanto cuantitativamente -por el número de víctimas y daños producidos- como cualitativamente -al incluir, junto a, o además de los tuaregs, a grupos terroristas islamistas, presentes en la región del Sahel desde algunos años antes, lo que convertía a Mali en un conflicto interno con claras consecuencias regionales e internacionales $-{ }^{6}$.

3 Sobre el principio uti possidetis iuris y su aplicación en el proceso de descolonización de los Estados africanos, vid., entre otros, Brownlie, I., African Boundaries. A legal and diplomatic Encyclopedia, C. Hurst \& Co. Publishers, London, 1979; CorTen, O., «Droit des peoples a disposer d'eux-mêmes et uti possidetis: deux faces d'une même médaille?», 31 Revue Belge de Droit Internationale, 161-189 (1998); Corten, O.; Delcourt, B.; Klein, P. y Levrat, N. (ed.), Démembrements d'Etats et délimitations territoriales : l' uti possidetis iuris en question(s), Bruylant, Bruxelles, 1999; KLABBERS, J. y LEFEER, R., «Africa: Lost between self-determnation and uti possidetis», en Peoples and Minorityes in International Law, Brolmann, Lefeber y Zieck (ed.), Dordrecht, 1993, pp. 37-76; Lalonde, S., Determining Boundaries in a Conflict World. The Role of Uti Possidetis, McGill-Queen's University Press, Montreal-Kingston, 2002; RaTnER, S. R., «Drawing a better line: Uti possidetis and the borders of new States», 90 American fournal of International Law 590624 (1996); SÁNCHEZ RodríGUEZ, L. I., «L'uti possidetis et les effectivités dans les contentieux territoriaux et frontaliers», 263 Collected Courses of the Hague Academy of International Law 1-379 (1997); YAKPO, E., «The African concept of uti possidetis - need for change?», en Liber Amicorum Fudge Mohammed Bedajoui, The Hague, 1999, 271-290.

4 Las regiones del Norte de Mali, sitas entre el Sahara y la faja del Sahel, están habitadas por pueblos árabes nómadas (Tuaregs, Fulani, Mora); en especial, los Tuaregs, pueblo homogéneo que, tras el proceso de descolonización, de la mano del mencionado principio uti possidetis y en flagrante contradicción -una de tantas en África- con el principio de libre determinación de los pueblos, quedó dividido entre varios Estados -Mali, Níger, Libia y Argelia-, cuyas fronteras en pleno Desierto del Sahara, más que porosas, nunca han existido para ellos. Mientras que el Sur de Mali, mucho más densamente poblado, se articula en torno a Bamako, la capital del Estado; región más propiamente subsahariana y con pueblos muy diferentes, entre sí y, sobre todo, en relación a los del Norte: Mande (Bambaras, Malinkes, Soninkes), Peul, Voltaic, entre otros. Vid. Mateos MartíneZ, M., «Étnias de Mali», <https://prezi.com/j5p3cnu0rkdb/etniasde-mali/> (para todas las webs citadas, última visita, 5 de septiembre de 2016).

5 Vid. Moraleda Martín-Peñato, M., «El pueblo tuareg y su papel en el conflicto de Mali», Documento de Opinión, Instituto Español de Estudios Estratégicos, 14 de agosto de 2013, <www. ieee.es/Galerias/fichero/docs_opinion/2013/DIEEEO75-2013_Tuaregs_MMoraleda.pdf>.

6 En especial para Europa, debido a su proximidad geográfica. Como ya señalaba Javier Solana en la Estrategia Europea de Seguridad de 2003 al analizar las diferentes amenazas para la Unión 
Así, la irrupción armada a partir del 17 de enero de 2012 del Movimiento Nacional por la Liberación de Azawad (MNLA) - movimiento nacionalista tuareg laico, que fue creado en Tombuctú en octubre de 2011 a partir de movimientos tuaregs de oposición al gobierno de Bamako y conformado por soldados tuaregs fuertemente armados, bregados en la guerra y muy capaces, provenientes de Libia una vez derrocado Gadafi ${ }^{-}$, de un lado; junto a la actuación, de otro y sin coordinación con el anterior, de Ansar Dine -grupo tuareg, pero de raíz yihadista salafista- y sus aliados, los grupos terroristas Al Qaeda en el Magreb Islámico (AQMI) y su escisión, el Movimiento para la Unidad de la Yibad en el África del Oeste (MUYAO), llevaron a la rápida conquista de las regiones del Norte y Centro de Mali. Así, entre el 30 de marzo y el 2 de abril de 2012 las regiones de Kidal, Gao y Tombuctú, con sus capitales, fueron ocupadas completamente por los rebeldes del MNLA y los grupos terroristas mencionados, que se enfrentaron entre sí, expulsando estos últimos al MNLA de las ciudades ${ }^{8}$.

El colapso del régimen de Bamako se completó con las desavenencias en el gobierno y ejército de Mali, que se tradujeron en el golpe de Estado de 22 de marzo de ese año. Con ello, la conversión de Mali en un verdadero Estado fallido y, más aún, el peligro cierto de la conquista completa del mismo por los grupos terroristas vinculados a $\mathrm{Al}$ Qaeda tomaba fuerza. Ello llevó a la intervención internacional que, tras no pocas dudas, acabó por tomar forma en enero de 2013 a través de la intervención militar de Francia-Operación Serval- que detuvo el avance de los grupos terroristas hacia Bamako y permitió la recuperación de las provincias de Tombuctú, Gao y Kidal en las semanas siguientes?.

Europea: «La unión de estos diferentes elementos -el terrorismo empeñado en ejercer la máxima violencia, la disponibilidad de armas de destrucción masiva, la delincuencia organizada, el debilitamiento del sistema estatal y la privatización de la fuerza- nos colocaría, sin duda, frente a una amenaza muy radical». Pues bien, salvo en lo relativo a las armas de destrucción masiva, en Mali se unieron el resto de las principales amenazas para Europa a partir de 2012, elevándolo así a la categoría de amenaza real muy radical. Estrategia Europea de Seguridad. Una Europa segura en un mundo mejor, Bruselas, 12 de diciembre de 2003, p. 5, <www.consilium.europa.eu/uedocs/ cmsUpload/031208ESSIIES.pdf>.

7 Recordemos que Muamar el Gadafi fue linchado hasta la muerte el 20 de octubre de 2011; lo que vino a marcar el final de la primera fase de la guerra civil en la que continúa sumida Libia.

8 The Office of the Prosecutor (ICC), Situation in Mali. Article 53(1) Report, 16 de enero de 2013, pp. 9-11.

9 Sobre la Operación Serval, vid. Barrera, B., Opération Serval: Notes de guerre, Mali 2013, Seuil, Paris, 2015; DíEz AlCALDE, J., «Mali: decisiva y contundente reacción militar de Francia para frenar el avance yihadista», Documento de análisis, Instituto Español de Estudios Estratégicos (IEEE), 23 de enero de 2013, <www.ieee.es/Galerias/fichero/docs_analisis/2013/ 
Sin embargo, a lo largo de los meses de 2012 en que Tombuctú, Gao y Kidal estuvieron en manos de los grupos salafistas Ansar Dine, AQMI y MUYAO sus líderes procedieron a decidir, ordenar y llevar a cabo la destrucción de gran parte del patrimonio cultural y religioso de dichas ciudades, en gran parte declarados Patrimonio de la Humanidad por la UNESCO. Así, Tombuctú fue incluida en la Lista del Patrimonio Mundial en $1988^{10}$, mientras que la Tumba de Askia, sita en Gao, lo fue en $2004^{11}$.

DIEEEA06-2013_Reaccion_de_Francia_en_Mali_JDA.pdf>; SHurkin, M., France's War in Mali Lessons for an Expeditionary Army, Rand Corporation, 2014, <www.rand.org/content/dam/ rand/pubs/research_reports/RR700/RR770/RAND_RR770.pdf $>$.

10 Como se indica en la Declaración de su excepcional valor universal: «Located at the gateway to the Sahara desert, within the confines of the fertile zone of the Sudan and in an exceptionally propitious site near to the river, Timbuktu is one of the cities of Africa whose name is the most heavily charged with history. Founded in the 5th century, the economic and cultural apogee of Timbuktu came about during the15th and 16th centuries. It was an important centre for the diffusion of Islamic culture with the University of Sankore, with 180 Koranic schools and 25,000 students. It was also a crossroads and an important market place where the trading of manuscripts was negotiated, and salt from Teghaza in the north, gold was sold, and cattle and grain from the south. (...) The three big Mosques of Djingareyber, Sankore and Sidi Yahia, sixteen mausoleums and holy public places, still bear witness to this prestigious past. The mosques are exceptional examples of earthen architecture and of traditional maintenance techniques, which continue to the present time. Criterion (ii): The mosques and holy places of Timbuktu have played an essential role in the spread of Islam in Africa at an early period. Criterion (iv): The three great mosques of Timbuktu, restored by the Qadi Al Aqib in the 16th century, bear witness to the golden age of the intellectual and spiritual capital at the end of the Askia dynasty. Criterion (v): The three mosques and mausoleums are outstanding witnesses to the urban establishment of Timbuktu, its important role of commercial, spiritual and cultural centre on the southern trans-Saharan trading route, and its traditional characteristic construction techniques. Their environment has now become very vulnerable under the impact of irreversible change», <http://whc.unesco.org/en/list/119>.

11 Según la Declaración de su excepcional valor universal: «The Tomb of Askia is located in the town of Gao. The site comprises the following elements: the pyramidal tower, the two flat-roofed mosques, the necropolis and the white stone square. The spectacular pyramidal structure was built by Askia Mohamed, Emperor of the Songhai Empire in 1495. The Tomb of Askia was built when Gao became the capital of the Empire and Islam was adopted as the official religion.

The Tomb of Askia is a magnificent example of how the local traditions have adapted to the exigences of Islam in creating an architectural structure unique across the West African Sahel. The Tomb is the most important and best conserved vestige of the powerful and rich Songhai Empire that extended through West Africa in the 15th and 16th centuries. Its value is also invested in its architectural tomb/minaret shape, its prayer rooms, its cemetery and its assembly space that have survived and are still in use. From the architectural perspective, the Tomb of Askia is an eminent example of Sudano-Sahelian style, characterized by rounded forms resulting in the regular renewal of the layer of plaster eroded each winter by the rare but violent rains. The pyramidal form of the tomb, its function as central minaret as well as the length and shape of the pieces of wood comprising the permanent scaffolding, give the Tomb of Askia its distinctive and unique architectural characteristics. 
Se trataba de un nuevo caso, uno más, de la política -en el sentido propio del elemento incluido en el tipo de crimen de lesa humanidad ${ }^{12}$, sólo que aquí los destinatarios directos del ataque no son la población civil sino los bienes culturales- de destrucción del patrimonio que, iniciada en marzo de 2001 por los talibanes en Afganistán con la destrucción, entre otros bienes culturales, de los conocidos Budas de Bamiyán, ha venido repitiéndose allá donde los grupos armados fundamentalistas islamistas lograban tomar el control efectivo de un territorio con bienes culturales y/o religiosos: Mali en 2012, Siria, Irak y Libia a partir de la autoproclamación del Estado Islámico en 2014.

Política-que no actuación irreflexiva, meramente refleja o compulsiva, ni nihilista- pensada y aplicada de forma sistemática con una lógica clara y bien definida que responde a la voluntad de acabar con los bienes culturales y/o religiosos ajenos y/o desviados de la interpretación fundamentalista-salafista del Islam que tienen dichos grupos suníes wahabíes ${ }^{13}$; calificándolos como in-

Criterion (ii): The Tomb of Askia reflects the way the local building traditions, in response to Islamic needs, absorbed influences from North Africa to create a unique architectural style across the West African Sahel. Criterion (iii): The Tomb of Askia is an important vestige of the Songhai Empire, which once dominated the Sahel lands of West Africa and controlled the lucrative trans-Saharan trade. Criterion (iv): The Tomb of Askia reflects the distinctive architectural tradition of the West African Sahel and in particular the way buildings evolve over centuries through regular, traditional maintenance practices», <http://whc.unesco.org/en/list/1139>.

12 De acuerdo con lo establecido en el artículo 7.2.a) del Estatuto de la Corte Penal Internacional: «Por 'ataque contra una población civil' se entenderá una línea de conducta que implique la comisión múltiple de actos mencionados en el párrafo 1 contra una población civil, de conformidad con la política de un Estado o de una organización de cometer ese ataque o para promover esa política». Cursiva añadidas.

13 Es importante tratar de ser lo más escrupuloso y exacto posible a la hora de identificar estos grupos, y la ideología que los alimenta y con base a la cual actúan, porque cualquier generalización en este sentido no sólo resulta inexacta, sino profundamente injusta y genera no pocos efectos negativos graves.

Para la distinción entre las distintas ramas del Islam y las fuentes o bases ideológicas de los grupos terroristas islamistas, vid., entre otros, ARMASTRONG, K., Los orígenes del fundamentalismo en el judaísmo, el cristianismo y el islam, Fabula Tusquets, Barcelona, 2004; BucK-Morss, Pensar tras el terror. El islamismo y la teoría crítica entre la izquierda, Antonio Machado Libros, Madrid, 2010; Gómez García, L., Diccionario de Islam e islamismo, Espasa, Madrid, 2009; GonZÁlez HERNÁNDEZ, M., «Definiendo conceptos: sufismo, fundamentalismo, salafismo, islamismo y wahabismo», en F. Vacas Fernández (dir.), Comunidad Internacional, Oriente Próximo y Cultura de $P a z, \mathrm{n}^{\circ} 3$ Cuadernos Cultura de Paz, Instituto de Estudios Internacionales y Europeos «Francisco de Vitoria» de la Universidad Carlos III de Madrid, 2015, pp. 57-68; Mosterín, J., El Islam, Alianza Editorial, Madrid, 2012; RichARD, Y., El Islam Shii, Biblioteca del Islam Contemporáneo, Ediciones Bellaterra, Barcelona, 1996; TAMAYO, J. J., Islam, cultura, religión y política, Trotta, Madrid, 2010; TAMAYO, J. J., «Pluralismo religioso en el Mundo Árabe», en F. Vacas Fernández (dir.), El Mundo Árabe hoy: entre inmovilismo y transformación, $\mathrm{n}^{\circ} 2$ Cuadernos Cultura de Paz, Instituto de Estudios Internacionales y europeos «Francisco de Vitoria» de la Universidad Carlos III de Madrid, 2014, pp. 23-32. 
fieles, los primeros, o heréticos, los segundos. Con ello, tratan de imponer su visión a las futuras generaciones, borrando el pasado -Budas de Bamiyán, Palmira...- o, incluso, como en Tombuctú, el presente cultural y religioso de los pueblos que dominan ${ }^{14}$ con la intención de imponer por la fuerza su ideología y dominar la población ${ }^{15}$.

$\mathrm{Y}$ es que el patrimonio, los bienes culturales y/o religiosos no son meros objetos materiales con un mayor o menor valor económico, un lujo a ser preservado únicamente para el disfrute del turismo cultural. En palabras de Fatou BEnsouda, Fiscal de la Corte Penal Internacional:

«Culture is who we are. Our ancestors created paintings, sculptures, mosques, temples and other forms of cultural possessions all around us. They put their hearts and souls into the creation of such cultural heritage so that it represents the cultural identity of their times, and is passed on for the benefit of future generations.

Indeed, this cultural heritage shapes the spirit and identity of our own generation and of generations to come. With the passage of time, they become

14 En palabras del antropólogo HuYsECOM: «Il est important pour moi de souligner qu'au Mali la situation est particulièrement tragique et distincte de la destruction des Bouddhas de Bamiyan en Afghanistan. A Bamiyan, il s'agissait de destructions terribles d'un patrimoine mondial pluri séculaire et exceptionnel, toutefois déconnecté de la croyance des populations locales (même si d'autres nations bouddhistes y étaient bien entendu religieusement attachées comme le Japon etc.). Au Nord-Mali, il s'agit non seulement de la destruction d'un patrimoine mondial, mais aussi, avant tout du patrimoine malien, du patrimoine des populations habitant Tombouctou. Impuissantes, elles assistent à la destruction, sous leurs yeux, des tombeaux de leurs ancêtres. De lieux saints toujours profondément vénérés». HuYsECOM, E., «Tombouctou: la destruction des mausolées par les islamistes d'Ansar Dine», Sciences et Avenir, 1 de juillet 2012, $<$ www.sciencesetavenir.fr/decryptage/20120701.OBS5726/tombouctou-la-destruction-desmausolees-par-les-islamistes-d-ansar-dine.html>.

15 Como recuerda Fatou BENSOUDA, «the mausoleums of Timbuktu played and continue to play an important religious role in the daily lives of the city's inhabitants. Mausoleums are sacred places of worship. They are frequently visited by the city's residents. Pilgrims would also come from distant places to pay their respects and to pray. Going to the mausoleums was and still is an expression of one's faith and religious piety.

It is specifically these deeply rooted religious practices and beliefs that Ansar Dine and AQIM wanted to annihilate by destroying these mausoleums. Through their brutal and callous acts, they made it impossible for the inhabitants of Timbuktu to devote themselves to their religious practices during the ten-month occupation of their city (...) To destroy Timbuktu's mausoleums is therefore to erase an element of collective identity built through the ages. It is to eradicate a civilisation's landmark. It is the destruction of the roots of an entire people, which irremediably affects its social attitudes, practices and structures». Statement of the Prosecutor of the International Criminal Court, Fatou Bensouda, at the opening of Trial in the case against Mr Abmad Al-Faqi Al Mahdi, 22 August 2016, pp. 3 y 4. Cursivas añadidas, <www.icc-cpi.int//Pages/item. aspx? name $=$ otp-stat-al-mahdi-160822>. 
archetypes of social memory from which individuals shape their identity and grow. (...) our cultural heritage is not a luxury good. Our cultural heritage is a vital instrument of human development.

To protect cultural property is to protect our culture, our history, our identity, and our ways of expressing faith and practicing religion for current and future generations. We must protect our common heritage from desecration, ravages and the long-term effects of such destructive acts» ${ }^{16}$.

Pero es que, además, los efectos negativos de su destrucción no se detiene en lo indicado, por grave y decisivo que sea; sino que, como afirma la UNESCO en su Estrategia para el refuerzo de la labor de la UNESCO en materia de protección de la cultura y promoción del pluralismo cultural en caso de conflicto armado: «El aumento de los ataques deliberados contra el patrimonio cultural registrado en los últimos diez años ha estado ligado a una estrategia de extremismo violento que utiliza la destrucción deliberada y sistemática de la cultura como arma de guerra (...). Esta forma de denegación polifacética de la cultura y la diversidad cultural (...) también pone de manifiesto que la destrucción del patrimonio cultural es mucho más que una tragedia cultural y ha pasado a convertirse en una cuestión de seguridad ${ }^{17}$, y por qué la protección de la cultura no se puede desvincular de las operaciones bumanitarias y ba de ser un elemento clave de cualquier estrategia de paz» ${ }^{18}$.

Por todo ello, para evitar la destrucción del patrimonio cultural y religioso de un pueblo -que es, además y por ello, de toda la Humanidad-, y con ello del pueblo mismo, pero también para consolidar la paz futura en situa-

$16 \mathrm{Ibid}, \mathrm{pp} .4$ y 7.

17 Como también ha subrayado Bensouda: «This type of crime, wherever it may happen in the world, presents the international community today with enormous challenges. (...) I would like to highlight three general points in this regard: The first is that deliberate attacks on cultural property are often the precursor to the worst outrages against a population. As some learned observers have noted, the struggle to defend the cultural property of a population is an integral part of the humanitarian operation aimed at protecting that population. The second aspect, one of greater concern, is that deliberate attacks on cultural property have become actual weapons of war. They are being used to eliminate entire communities and wipe out any traces left of them, their history and identity, as though they never existed. (...) Lastly, the protection of cultural heritage is an essential part of the post-conflict social reconstruction and reconciliation process. This is because cultural heritage gives meaning as well as a sense of continuity and direction from the past to the future». Ibid, pp. 5 y 6.

18 Refuerzo de la labor de la UNESCO en materia de protección de la cultura y promoción del pluralismo cultural en caso de conflicto armado, doc. $38 \mathrm{C} / 49$, de 30 de octubre de 2015, par. 1 (accesible en <http://unesdoc.unesco.org/images/0023/002351/235186s.pdf>). 
ciones de conflicto armado, la Comunidad Internacional ha tratado de actuar a favor de la preservación del patrimonio cultural, en especial, en conflictos armados, cuando más expuesto está. Sin embargo, debemos reconocer que los resultados hasta el momento no han sido los deseables. Es más, como hemos indicado, los bienes culturales están pasando a ser considerados por algunos grupos en armas como objetivos en sí mismos, a destruir dentro de su perversa lógica de imposición ideológico-religiosa, que bien podríamos calificar, con Irina BoKOVA, como una verdadera 'limpieza cultural' ${ }^{19}$.

Es precisamente en este descorazonador contexto en el que, por primera vez, se ha producido la actuación concertada y decidida de la Comunidad Internacional para la protección del patrimonio cultural en un país en situación de conflicto armado: en Mali el Consejo de Seguridad de las Naciones Unidas, la UNESCO y la Corte Penal Internacional han mostrando su capacidad de responder a los graves desafíos que en esta materia afronta la Humanidad y han demostrado que con voluntad política y actuación conjunta y coordinada se puede proteger, reconstruir y conservar eficazmente el patrimonio de un pueblo; que es proteger, reconstruir y conservar la identidad del pueblo mismo y, en definitiva, de la paz de las personas que lo conforman.

\section{La aCtuación de las Naciones Unidas: La misión de las NaCiOneS UNIDAS EN MALI (MINUSMA), PRIMERA OPERACIÓN DE PAZ EN CUYO mandato Se incluye la Protección del Patrimonio Cultural}

Puede resultar sorprendente constatar que la Misión de las Naciones Unidas en Mali (MINUSMA), establecida por el Consejo de Seguridad en abril de 2013, sea la primera, en la larga historia de las operaciones de paz de las Naciones Unidas, en la que se hace expresa referencia a la protección del patrimonio cultural del Estado en el que se despliega. Aunque quizás no lo sea tanto si se tiene en cuenta la importancia muy relativa que ha venido teniendo para los ejércitos, y para los Estados mismos, la salvaguardia real y efectiva - más acá de las grandilocuentes palabras de convenciones, declaraciones o discursos- del patrimonio y los bienes culturales en conflictos armados; mucho

19 Expresión utilizada por primera vez por la Directora General de la UNESCO, Irina BoKOVA, en agosto de 2014. Vid. <www.unesco.org/new/es/media-services/single-view/news/preserving_the_cultural_heritage_and_diversity_of_syria_and_iraq_are_essential_irina_bokova_welcomes_at_unesco_un_deputy_secretary_general_jan_eliasson/\#.V-JLKPmLTcs>. 
más centrados en las lógica bélica para ganar la guerra u obtener una ventaja militar específica ${ }^{20}$. Y es que, como subraya KILA: «The military do not consider knowledge and handling of cultural goods and environments part of their core-business and certainly not a priority (...). When mentioning the subject in military organizations cultural heritage is considered to have low priority and often the communication stops at this point $\gg^{21}$.

Y ello tanto en relación a la conducción de operaciones bélicas unilaterales como en el marco de operaciones multilaterales, ya en coalición ya bajo el paraguas de una organización internacional. Así, como subrayan RoDRÍGUEZ TERMiÑo y GONZÁLEZ ACUÑA en relación a la participación de expertos en la protección del patrimonio cultural en unidades de ciertas operaciones militares internacionales: «Con independencia de la cuestionable utilidad del conocido como Human Terrain System (HTS), en los casos de Afganistán e Irak, debido sobre todo a errores de escala y medios, nos preocupa cómo en la práctica se cuestionan los fundamentos éticos de los profesionales que asesoran en estos temas. Tensión producida por la incompatibilidad entre las prioridades militares sobre el terreno, básicamente evitar bajas entre los soldados y aumentar la eficacia de las misiones bélicas, y la deontología científica, lo que convierte el objetivo de ganar los corazones y las mentes de la población local en un mero eslogan sin aplicación práctica» ${ }^{22}$.

20 Baste recordar el fracaso de buena parte de los mecanismos de protección y salvaguardia previstos en la Convención de La Haya de 1954, simplemente por su no utilización por los Estados al priorizar la «lógica de la guerra» a la de la salvaguardia de los bienes culturales; lo que llevó a la adopción del Segundo Protocolo a la Convención de La Haya para la protección de bienes culturales en caso de conflicto armado, de 26 de marzo de 1999. Como se indica en su Preámbulo: «Conscientes de la necesidad de mejorar la protección de los bienes culturales en caso de conflicto armado y de establecer un sistema reforzado de protección para bienes culturales especialmente designados; Reiterando la importancia de las disposiciones de la Convención para la Protección de los Bienes Culturales en caso de Conflicto Armado adoptada en La Haya el 14 de mayo de 1954, y haciendo hincapié en la necesidad de completar esas disposiciones con medidas que refuercen su aplicación; Deseosas de proporcionar a las Altas Partes Contratantes en la Convención un medio para participar más estrechamente en la protección de los bienes culturales en caso de conflicto armado mediante el establecimiento de procedimientos adecuados».

21 KILA, J. D., «Utilizing Military Cultural Experts in Times of War and Peace: An Introduction», en Culture and international Law, P. Meerts (ed.), Hague Academic Coalition, The Hague, 2008, p. 190.

22 Rodríguez Termiño, I. y GonZÁlez AcuÑa, D., «La protección del patrimonio cultural en conflictos armados. De las lecciones aprendidas al diseño estratégico», Instituto Español de Estudios Estratégicos (IEEE), Documento Marco, 15/2013, de 7 de agosto de 2013, p. 7, <www. ieee.es/Galerias/fichero/docs_marco/2013/DIEEEM15-2013_ProteccionPatrimonioCulturasConflictosArmados_IRguez.pdf>. 
Sin embargo, la salvaguardia del patrimonio cultural de los pueblos donde este tipo de operaciones militares se despliegan no implica sólo, ni siquiera principalmente, una cuestión de imagen, de relaciones públicas dirigida a ganar los corazones y las mentes de la población local; por más que ello sea deseable desde la perspectiva de la eficacia de la operación. En muchos de los conflictos actuales, como hemos señalado, el patrimonio cultural de los pueblos forma parte de la guerra misma y, por consiguiente, es clave en la victoria o la derrota: puesto que el patrimonio cultural de los pueblos es realmente considerado en la lógica de, al menos, uno de los contendientes -los grupos armados islamistas en Afganistán, Irak, Siria, Mali o Libia- como objetivo político-militar mayor y como tal tratan de destruirlo; su salvaguardia y protección -y, en su caso, su restauración- deben dejar de ser entendidas por los Estados y sus ejércitos únicamente como un objetivo ético-jurídico abstracto -por más que loable y bienintencionado, pero en todo caso susceptible de ser sacrificado ante objetivos militares específicos-, para pasar a ser considerado también como un objetivo militar concreto, de cuya consecución depende en gran medida la victoria en el conflicto; así como la consolidación de la paz posterior y la prevención de nuevos conflictos, no lo olvidemos.

Por otra parte, esa misma minusvaloración de la importancia del patrimonio cultural ha llevado en no pocas ocasiones a que desde esas mismas operaciones internacionales se haya contribuido a dañar dicho patrimonio ya de forma pasiva -no adoptando las, posibles y necesarias, medidas para protegerlo-, ya por la actuación de algunos de sus componentes. Como recuerdan RODRíGUEZ TERMiÑo y GONZÁLEZ ACUÑA: «Las más recientes intervenciones militares internacionales, auspiciadas o no por resoluciones del Consejo de Seguridad de la ONU, tampoco se libran de actuaciones que han supuesto un daño irreparable al patrimonio de las zonas afectadas, generalmente por la ausencia de interés y de una mínima preparación previa al despliegue sobre el terreno» ${ }^{23}$. Así, por ejemplo, las destrucciones acaecidas en Babilonia, Ur o Samarra por parte de las tropas de la Coalición internacional liderada por Estados Unidos en Irak en $2003^{24}$ o las recientes destrucciones del patrimo-

$23 \mathrm{Ibid}$, p. 5.

24 Vid. UNESCO, International Coordination Committee for the Saveguarding of the Cultural Heritage of Iraq: Final Report on Damage Assessments in Babylon, doc. CLT/EO/CIP/2009/RP/114, 2009, <www.unesco.org/fileadmin/MULTIMEDIA/FIELD/Iraq/pdf/Report\%20on\%20Damages\%20in\%20Babylon.pdf>. 
nio cultural del Yemen por parte de la coalición militar liderada por Arabia Saudín.

Pues bien, tanto para un caso como para el otro, la MINUSMA ha supuesto un punto de inflexión al incorporar el Consejo de Seguridad, por primera vez en el marco de una operación de paz de Naciones Unidas, de un lado, la función de «Apoyo a la preservación del patrimonio cultural» dentro de su mandato, y, por otro, recordando en sus resoluciones la obligación de respetar y hacer todo lo posible para salvaguardar dicho patrimonio en el marco del desarrollo de la operación, por parte de la operación misma y por sus componentes.

En efecto, por lo que respecta al mandato, la resolución 2100 (2013), de 25 de abril, que establece la MINUSMA, ha marcado una primicia en la acción de la Comunidad Internacional en la protección del patrimonio cultural en conflictos armados, al incluir en su mandato la función de «Apoyo a la preservación del patrimonio cultural», junto a la, también por primera vez, de «Apoyo a la justicia nacional e internacional»; para lo cual, además, el Consejo de Seguridad le autoriza el uso coercitivo de la fuerza:

«16. Decide que el mandato de la MINUSMA será el siguiente:

(...)

f) Ayudar a las autoridades de transición de Malí, cuando sea necesario y viable, a proteger contra posibles ataques los lugares de importancia cultural e histórica de Malí, en colaboración con la UNESCO;

g) Prestar apoyo, cuando sea viable y apropiado, a los esfuerzos que realicen las autoridades de transición de Malí, sin perjuicio de sus responsabilidades, para detener y llevar ante la justicia a los responsables de los crímenes de guerra y crímenes de lesa humanidad cometidos en Malí, teniendo en cuenta la remisión por las autoridades de transición de Malí de la situación imperante en su país desde enero de 2012 a la Corte Penal Internacional;

25 World Heritage Committee, Walled City of Sana'a (Yemen), Decision 40 COM 7A.24, 2016, $<$ http://whc.unesco.org/en/decisions/6639>. Vid. también La Directora General de la UNESCO condena la destrucción de los edificios históricos de la Ciudad Vieja de Saná, en Yemen (accesible en $<$ http://es.unesco.org/news/directora-general-unesco-condena-destruccion-edificios-historicos-ciudad-vieja-sana-yemen>); La Directora General de la UNESCO pide a todas las partes que protejan el patrimonio cultural de Yemen, 12 de mayo de 2015, <www.unesco.org/new/es/media-services/single-view/news/la_directora_general_de_la_unesco_pide_a_todas_las_partes_ que_protejan_el_patrimonio_cultural_de_yemen/\#.V9gKg_mLTcs $>$. 
17. Autoriza a la MINUSMA a que utilice todos los medios necesarios, dentro de los límites de su capacidad y zonas de despliegue, para llevar a cabo su mandato enunciado en los párrafos 16 a) i) y ii), 16 c) i) y iii), 16 e), $16 \mathrm{f)}$ y $16 \mathrm{~g}) \gg^{26}$.

Resulta pertinente destacar no sólo la novedosa incorporación a un mandato de una operación de paz de las Naciones Unidas de ambas funciones, sino también su formulación seguida en el mandato, que implica una cierta conexión entre ambas que, como veremos en el tercero apartado, se ha plasmado en la realidad y que muestra bien la necesidad de la trasversalidad y, muy unido a ello, la coordinación de los diferentes mecanismos que la Comunidad Internacional dispone para la salvaguardia, protección y recuperación del patrimonio cultural amenazado y/o efectivamente dañado en un conflicto armado.

Junto a ello, la autorización del uso coercitivo de la fuerza por parte del Consejo de Seguridad para hacer cumplir ambas funciones a través de la consabida fórmula «autoriza a la MINUSMA a que utilice todos los medios necesarios para» no hace sino subrayar, de un lado, la importancia que el propio Consejo da a dichas funciones y, sobre todo, la íntima conexión existente entre ellas y el mantenimiento de la paz y la seguridad internacionales o, dicho de otra manera -más apegada a la letra y espíritu del sistema de seguridad colectiva diseñado en el capítulo VII de la Carta de Naciones Unidas-, la amenaza que a la paz y la seguridad internacionales supone la destrucción del patrimonio cultural de los pueblos, base jurídica necesaria para la autorización del uso coercitivo de la fuerza en nuestro sistema, como bien sabemos ${ }^{27}$.

En cuanto a la obligación de respetar y hacer todo lo posible para salvaguardar dicho patrimonio, ésta recae en los Estados tal y como establecen el Convenio de La Haya para la protección de los bienes culturales en caso de conflicto armado, de 1954, tanto durante las operaciones bélicas -artículo 4- como en casos de ocupación militar -artículo 5-, y su Segundo Protocolo, de 1999 -artículos 7, 8 y $9 \_28$. Obligaciones jurídicas convencionales que, además, son parte del De-

26 S/RES/2100 (2013), de 25 de abril.

27 Vid. VACAS FERnáNDEZ, F., El régimen jurídico del uso de la fuerza por parte de las operaciones de mantenimiento de la paz de Naciones Unidas, Marcial Pons, Madrid, 2005.

28 Obligaciones de los Estados que reitera, en 2003, la Declaración de la UNESCO relativa a la destrucción intencional del patrimonio cultural, sea o no en el marco de un conflicto armado. Declaración de la UNESCO relativa a la destrucción intencional del patrimonio cultural, 17 de octubre de 2003, <http://portal.unesco.org/es/ev.php-URL_ID=17718\&URL_DO=DO_TOPIC\&URL_ SECTION=201.html $>$. 
recho Internacional general al ser Derecho consuetudinario ${ }^{29}$. Pues bien, resulta evidente que en el nivel operativo el cumplimiento de tales obligaciones recae en los ejércitos de los Estados, ya actuando como tales ya como unidades militares adscritas a operaciones bajo autoridad internacional ${ }^{30}$.

En este último caso, y por lo que a las operaciones de paz de Naciones Unidas respecta, el Boletín del Secretario General de 1999 titulado Observancia del derecho internacional humanitario por las fuerzas de las Naciones Unidas, expresamente establece:

«Se prohíbe a la fuerza de las Naciones Unidas atacar monumentos artísticos, arquitectónicos o históricos, lugares arqueológicos, obras de arte, lugares de culto y museos y bibliotecas que constituyan el patrimonio cultural o espiritual de los pueblos. La fuerza de las Naciones Unidas, en su zona de operaciones, no utilizará este patrimonio cultural o sus alrededores para fines que puedan exponerlo a sufrir daños o destrucción. Quedan

29 Sobre la protección jurídica internacional del patrimonio cultural en conflictos armados vid., entre otros, FERnández LiESA, C. R., «El Convenio de protección de los bienes culturales en caso de conflicto armado de 1954, y los Protocolos de 1954 y 1999», en C. R. Fernández Liesa y J. Prieto de Pedro (dir.), La protección jurídico internacional del patrimonio cultural, Colex, Majadahonda, 2009, pp. 103-133; GARCía LABAJO, J. M., «La protección de bienes culturales en caso de conflicto armado», en J. L. Rodríguez-Villasante y Prieto (coord.), Derecho Internacional Humanitario, $2^{a}$ ed., Tirant lo Blanch, Valencia, 2007, pp. 681-709; HenCKAERTs, J.-M., «Nuevas normas para la protección de los bienes culturales durante un conflicto armado», Revista Internacional de la Cruz Roja (30 de septiembre de 1999), <www.icrc.org/spa/resources/documents/misc/5tdnnb.htm ; NAHLIK, S. E., «La protection internationale des biens culturels en cas de conflict armé», 120 R.C.A.D.I. 61 (1967); NAHLIK, S.-T., «Protection of Cultural Property», en Les dimensions internationales du droit humanitaire, UNESCO/HenryDunant Institut/Martinus Nijhoff Publishers (ed.), Paris/Geneva/London, 1988; STAVRAKI, E., La Convention pour la protection des biens culturels en cas de conflit armé. Une Convention de droit international humanitaire, ed. Ant. N. Sakkoulas, Athènes-Komotini, 1996; Toman, J., La protection des biens culturels en cas de conflit armé. Commentaire de la Convention de La Haye du 14 Mai 1954 pour la protection des biens culturels en cas de conflit armé ainsi que d'autres instruments de droit international relatifs à cette protection, Paris, UNESCO, 1994; VERRI, P., «The condition of cultural property in armed conflicts (II)», en International Review of the Red Cross, Geneva, $\mathrm{n}^{\circ} 246$ (mayo-junio 1985).

30 En palabras de KILA: «In the field of Cultural Property protection, the military are a major player. They not only have the state monopoly on the use of force when operating in their national territory, but also in certain cases through mandates and joint missions, restricted by rules of engagement, in areas outside their country of origin. Soldiers operate during all phases of a conflict including those in which civil experts are not allowed or unable to function. In fact, they form both part of the problem and of the solution». KILA, J. D., Heritage under siege: military implementation of the 1954 Convention for the Protection of Cultural Property, $\mathrm{PhD}$ thesis, 2012, p. 38, $<$ http://dare.uva.nl/document/2/101238> y publicado con el mismo título en Brill, Leiden, 2012. Cursivas añadidas. 
terminantemente prohibidos el robo, el pillaje, la apropiación indebida y cualquier acto de vandalismo dirigido contra el patrimonio cultural» ${ }^{31}$.

Y aunque dichas obligaciones se supeditan al principio de necesidad militar, recogido en el párrafo segundo del artículo 5 del Convenio de La Haya de 1954 , por más que definido de forma muy estricta ${ }^{32}$, y limitado todavía más en el artículo 6 del Protocolo $\mathrm{II}^{33}$; la afirmación del General EISENHOWER realizada el 29 de diciembre de 1943 todavía tiene validez, sobre todo en los oídos militares, en toda su condicionada extensión: «We are bound to respct monuments as far as war allows. If we have to choose between destroying a famous building and sacrificing our men, then our men's lives count infinitely more and the buildings must go. But the choice is not always so clear-cut as that. In many cases the monuments can be spared without detriment to operational needs. Nothing can stand against the argument of military necessity but the phrase is sometimes used where it would be more truthful to speak of military or even personal convenience» ${ }^{34}$.

31 Sección 6.6 del Boletín del Secretario General, Observancia del derecho internacional humanitario por las fuerzas de las Naciones Unidas, ST/SGB/1999/13, de 6 de agosto de 1999, <www.un.org/ es/comun/docs/?symbol=ST/SGB/1999/13>.

32 «Las obligaciones definidas en el párrafo primero del presente artículo no podrán dejar de cumplirse más que en el caso de que una necesidad militar impida de manera imperativa su cumplimiento» (cursivas añadidas).

33 «A fin de garantizar el respeto de los bienes culturales de conformidad con el artículo 4 de la Convención:

(a) una derogación fundada en una necesidad militar imperativa conforme al párrafo 2 del artículo 4 de la Convención sólo se podrá invocar para dirigir un acto de hostilidad contra un bien cultural cuando y durante todo el tiempo en que:

i) ese bien cultural, por su función, haya sido transformado en un objetivo militar; y

ii) no exista otra alternativa prácticamente posible para obtener una ventaja militar equiva-

lente a la que ofrece el hecho de dirigir un acto de hostilidad contra ese objetivo;

b) una derogación fundada en una necesidad militar imperativa conforme al párrafo 2 del artículo 4 de la Convención sólo se podrá invocar para utilizar bienes culturales con una finalidad que pueda exponerles a la destrucción o al deterioro cuando y durante todo el tiempo en que resulte imposible elegir entre esa utilización de los bienes culturales y otro método factible para obtener una ventaja militar equivalente;

c) la decisión de invocar una necesidad militar imperativa solamente será tomada por el oficial que mande una fuerza de dimensión igual o superior a la de un batallón, o de menor dimensión cuando las circunstancias no permitan actuar de otra manera;

d) en caso de ataque basado en una decisión tomada de conformidad con el apartado a) se debe dará aviso con la debida antelación y por medios eficaces, siempre y cuando las circunstancias lo permitan».

34 Citado en KILA, J. D., «Utilizing Military Cultural Experts...», op. cit., p. 183. 
Pues bien, para la mejor y más efectiva aplicación de las mencionadas obligaciones, el Convenio de La Haya de 1954 establece en su artículo 7 los siguientes compromisos de los Estados:

«1. Las Altas Partes Contratantes se comprometen a introducir en tiempo de paz en los reglamentos u ordenanzas para uso de sus tropas, disposiciones encaminadas a asegurar la observancia de la presente Convención y a inculcar en el personal de sus fuerzas armadas un espíritu de respeto a la cultura y a los bienes culturales de todos los pueblos.

2. Se comprometen asimismo a preparar o establecer en tiempo de paz y en el seno de sus unidades militares, servicios o personal especializado cuya misión consista en velar por el respeto a los bienes culturales y colaborar con las autoridades civiles encargadas de la salvaguardia de dichos bienes».

Sin embargo, son pocos los ejércitos que han desarrollado dichos compromisos educativos de manera profunda y sistemática y muchos menos los que cuentan con personal o servicios especializados en la materia ${ }^{35}$; por más que sea un lugar común afirmar que «la mejor manera de prever desastres patrimoniales, como el Irak ${ }^{36}$, es trabajar con las tropas y educarlas en el respeto hacia el patrimonio cultural ${ }^{37}$. Los principales daños al patrimonio arqueológico iraquí no tuvieron lugar durante las operaciones bélicas, sino una vez concluidas, en la fase denominada de estabilización. Lo cual podría haberse evitado con un mayor entrenamiento prebélico de las fuerzas militares» ${ }^{38}$.

35 Vid. KILA, J. D., «Heritage under siege...», op. cit., pp. 14 y ss. y 243 y ss.

36 Sobre la destrucción del patrimonio cultural de Irak a partir de 2003, vid., entre otros, EMBERLING, G. y Hanson, K. (eds.). Catastrophe! The Looting and Destruction of Iraq's Past, The University of Chicago, Chicago, 2008; HeESE, M. vaN, «Iraq and the Second Protocol to the 1954 Hague Convention», en Protecting Cultural Property in Armed Conflict. An Insight into the 1999 Second Protocol to the Hague Convention of 1954 for the Protection of Cultural Property in the Event of Armed Conflict, N. van Woudenberg y L. Lijnzaad (ed.), Brill-Nijhoff, 2014, Chap. 13; RoTHFIELD, L. (ed.), Antiquities under Siege: Cultural Heritage Protection after the Iraq War: AltaMira Press, Lanham (MD), 2008; Rush, L. W., «Response to 'Relations between Archaeologists and the Military in the Case of Iraq'», Papers from the Institute of Archaeology 19 (2009); STONE, P. G. y BAJJALY, J. F., The Destruction of Cultural Heritage in Iraq, Boydell \& Brewer Ltd, Woodbridge, 2008.

37 Vid. Zeidler, J. y Rush, L. W. «In-Theatre Soldier Training through' Cultural Heritage Playing Cards: A US Department of Defense Example», en Archaeology, Cultural Property, and the Military, L. W. Rush (ed.), The Boydell Press, Woodbridge, 2010, p. 75; RusH, L. W., «Dealing the Heritage Hand: Establishing a United State Department of Defense Cultural Property Protection Program for Global Operations», en ibid, pp. 86-97.

38 RodrígueZ Termiño, I. y GONZÁLEZ ACUÑA, D., «La protección del patrimonio cultural en conflictos armados...», op. cit., p. 22. 
De ahí que sea todavía más relevante el contenido de las sucesivas resoluciones del Consejo de Seguridad sobre la MINUSMA a partir de la resolución 2164 (2014), de 25 de junio, en las que, dirigiéndose a la operación misma y sus componentes, les recuerda la importancia de salvaguardar el patrimonio de Mali:

«Solicita al Secretario General que considere el impacto ambiental de las operaciones que realice la MINUSMA al cumplir las tareas incluidas en su mandato y, en este contexto, alienta a la MINUSMA a que lo gestione, según proceda y de conformidad con las resoluciones de la Asamblea General y los reglamentos de las Naciones Unidas aplicables y pertinentes, y a que realice sus actividades de manera cuidadosa en las proximidades de sitios culturales e históricos» ${ }^{39}$.

Pues bien, como vamos a ver seguidamente, la estrecha colaboración de la UNESCO con la MINUSMA va a resultar esencial tanto para el cumplimiento de su mandato como para la preparación y sensibilización de los componentes de la operación en cumplimiento, en este caso, de sus obligaciones en esta materia; entre ellas, el desarrollo de sus actividades «de manera cuidadosa en las proximidades de sitios culturales e históricos».

\section{La aCtuación de la Unesco en MaLi}

El artículo I.2.c) de la Constitución de la UNESCO encomienda a la Organización que ayude a conservar, hacer progresar y difundir el saber «velando por la conservación y la protección del patrimonio universal de libros, obras de arte y monumentos de interés histórico o científico». Se trata de una competencia que la UNESCO puede, y debe ${ }^{40}$, desarrollar pos sí y en cooperación con los Estados y otras organizaciones internacionales, así como con la sociedad civil. Sobre esta base jurídica, la UNESCO ha venido desarrollando toda una serie de iniciativas en el ámbito de la salvaguardia de la diversidad

39 S/RES/2164 (2014), de 25 de junio. Cursivas añadidas. Reiterada en sus resoluciones 2227 (2015), de 29 de junio, par. 25; 2295 (2016), de 29 de junio, par. 39.

40 Recordemos que, en puridad jurídica, «competencia» hace referencia a un poder/deber; mientras que es la palabra «potestad» la que denota únicamente un poder que no lleva aparejado deber alguno. 
y el patrimonio cultural en conflictos $\operatorname{armados}^{41}$ : de un lado, declaraciones y proyectos generales; de otro, actividades y programas concretos de protección y reconstrucción ante situaciones específicas.

En relación a las primeras, destaca, junto a las ya citadas Declaración de la UNESCO relativa a la destrucción intencional del patrimonio cultural, de $2003^{42}$, y la Estrategia para reforzar la labor de la UNESCO en materia de protección de la cultura y promoción del pluralismo cultural en caso de conflicto armado, de 2015, la Coalición Mundial «Unite4Heritage $»^{43}$, puesta en marcha durante la $39^{a}$ reunión del Comité del Patrimonio Mundial, celebrada en Bonn en julio de $2015^{44}$. Como se puede observar, la profunda preocupación que despierta en la Comunidad Internacional el aumento de los ataques al patrimonio cultural en el presente siglo ha llevado a la UNESCO a reaccionar tratando de liderar la respuesta conjunta y coordinada de la Comunidad Internacional; y hacerlo a partir de la relativamente amplia ya experiencia acumulada por la Organización especialmente a lo largo de las dos últimas décadas.

En efecto, como se subraya en la Estrategia para reforzar la labor de la UNESCO en materia de protección de la cultura y promoción del pluralismo cultural en caso de conflicto armado: «El apoyo a procesos importantes de recuperación y reconstrucción después de los conflictos en el ámbito de la cultura, como por ejemplo en Camboya, los Balcanes, el Afganistán o Malí, ha dotado a la UNESCO de una gran variedad de conocimientos especializados, redes y buenas prácticas (...). La presente estrategia parte de las enseñanzas extraídas

41 Vid. Lizarazu Perinat, M. T., «El papel de la UNESCO en la protección del patrimonio en zonas de conflicto», Actualidad Furídica Uría Menéndez, 42-2016, pp. 7-16.

42 Adoptada como respuesta a la destrucción de los conocidos como Budas de Bamiyan.

$43<$ www.unite4heritage.org/es/node/3>.

44 La Coalición Mundial «Unidos por el Patrimonio» se ha creado con el objetivo de erigirse «como marco de reflexión permanente acerca del modo de incorporar la protección de la cultura a los ámbitos humanitario, de seguridad y de consolidación de la paz. En el ejercicio de su función de laboratorio de ideas, la UNESCO actuará como plataforma mundial para promover ideas, así como acciones concretas en este sentido». «Refuerzo de la labor de la UNESCO en materia de protección de la cultura...», op. cit., par. 32. Una de sus primeras materializaciones ha sido la creación por parte de Italia de una Fuerza de Emergencia para la Cultura -Italian Task Force for Culture- a través de un acuerdo con UNESCO adoptado el 16 de febrero de 2016. Memorandum of Understanding between the government of the Italian Republic and UNESCO on the Italian National «Task Force in the framework of UNESCO's Global Coalition Unite4Heritage» for initiatives in favor of Countries facing emergencies that may affect the protection and safeguarding of culture and the promotion of cultural pluralism, <www.beniculturali.it/mibac/multimedia/MiBAC/documents/1455616287505_2._Memorandum_of_Understanding__11_II_2016_DRAFT_Finale_UNESCO_versione_Italia.pdf $>$. 
de dichas experiencias con el fin de seguir mejorando la pertinencia, la calidad y la incidencia de las actividades que lleva a cabo la UNESCO para proteger la cultura $\gg^{45}$. Finalidad dirigida a alcanzar los dos objetivos interrelacionados de la UNESCO en esta materia: fortalecer la capacidad de los Estados Miembros para prevenir y mitigar las pérdidas de patrimonio y diversidad culturales derivadas de conflictos y para recuperarse de ellas, e incorporar la protección de la cultura a la acción humanitaria, las estrategias de seguridad y los procesos de consolidación de la paz, colaborando con las partes interesadas pertinentes ajenas al ámbito de la cultura ${ }^{46}$.

Pues bien, de todas esas experiencias, la labor de la UNESCO en Mali está resultando especialmente valiosa como ejemplo de aplicación a una situación específica de los instrumentos previstos -especialmente en relación al primer objetivo: el fortalecimiento de la capacidad de los Estados- y de ensayo de otros nuevos, nunca antes intentados, que abre camino y muestran, desde actuaciones concretas, buenas prácticas que podrán ser seguidas en otras situaciones de crisis -en especial, en relación con el segundo objetivo: incorporar la protección de la cultura a la acción humanitaria, las estrategias de seguridad y los procesos de consolidación de la paz-. En realidad, la labor desarrollada por la UNESCO desde 2013 en Mali, en estrecha colaboración con la MINUSMA y con el Gobierno de maliense, ha resultado decisiva a la hora de dotar de contenido y lanzar las estrategias y proyectos generales indicados, como la propia UNESCO reconoce en su resolución Refuerzo de la labor de la UNESCO en materia de protección de la cultura y promoción del pluralismo cultural en caso de conflicto armado, de noviembre de 2015:

«Apoya los esfuerzos de la Directora General destinados a incorporar la protección del patrimonio cultural y la diversidad cultural, según proceda, en la acción humanitaria, las estrategias globales de seguridad aprobadas por las $\mathrm{Na}$ ciones Unidas y los procesos de consolidación de la paz, por medio de todos los mecanismos pertinentes de las Naciones Unidas y en colaboración con los departamentos competentes de estas, tomando en cuenta los resultados positivos obtenidos por la Misión Multidimensional Integrada de Estabilización de las Naciones Unidas en Malí (MINUSMA)» $\gg^{47}$.

45 «Refuerzo de la labor de la UNESCO en materia de protección de la cultura...», op. cit., par. 11.

46 Ibid, par. 16.

47 Resolución UNESCO, Refuerzo de la labor de la UNESCO en materia de protección de la cultura y promoción del pluralismo cultural en caso de conflicto armado, doc. $38 \mathrm{C} / 48$, de 17 de noviembre de 2015, pto. 6, <http://unesdoc.unesco.org/images/0024/002433/243325s.pdf>. 
Para su análisis podemos distinguir dos grandes ámbitos: la labor de preparación y concienciación del personal de la MINUSMA para la protección y salvaguardia del patrimonio cultural de Mali, de un lado, y la reconstrucción del patrimonio cultural de Mali destruido o dañado en el conflicto, de otro.

\subsection{La labor de concienciación y capacitación del personal de la MINUSMA para la protección del patrimonio cultural de Mali}

Como hemos indicado, el artículo 7 del Convenio de La Haya de 1954 establece la obligación para los Estados de «inculcar en el personal de sus fuerzas armadas un espíritu de respeto a la cultura y a los bienes culturales de todos los pueblos»; así como de «preparar o establecer en tiempo de paz y en el seno de sus unidades militares, servicios o personal especializado cuya misión consista en velar por el respeto a los bienes culturales y colaborar con las autoridades civiles encargadas de la salvaguardia de dichos bienes».

A pesar de tales previsiones, que suponen obligaciones jurídicas positivas para los Estados, no debemos olvidar la ya referida ausencia de interés o minusvaloración del patrimonio cultural por parte de los ejércitos, reconociendo que la misma no tiene un responsable único sino que ha sido el resultado de carencias en diferentes ámbitos. Como ejemplifica KILA: «Many experts working for civilian cultural institutions like museums, non-governmental organizations (NGOs), and universities generally consider the military to be an entity that destroys culture, or in the best case, an organization not interested in cultural sites and goods whereas a military discussion about cultural activities during a mission may be labeled 'mission creep'. An ongoing dialogue between both parties is not only needed, but is of the utmost importance ${ }^{48}$.

De ahí que en 1999 se viera la necesidad de reiterar y especificar las obligaciones mencionadas de educación y sensibilización del personal militar en materia de respeto y protección de bienes culturales, en el capítulo 7 del Protocolo 2 al Convenio de La Haya, donde se subraya, de manera reiterada, el papel destacado que en este ámbito debe jugar la UNESCO. Y es que, en efecto, la labor de la UNESCO en materia de sensibilización y capacitación

48 KILA, J. D., «Utilizing Military Cultural Experts...», op. cit., p. 190. 
de las fuerzas armadas en esta materia resulta esencial, tal y como prevé su artículo 33, significativamente titulado «Asistencia de la UNESCO»:

«1. Las Partes podrán recurrir a la asistencia técnica de la UNESCO para organizar la protección de sus bienes culturales, especialmente en relación con medidas preparatorias para salvaguardar bienes culturales y con medidas preventivas y organizativas para situaciones de emergencia y realización de catálogos nacionales de bienes culturales, o en relación con cualquier otro problema derivado de la aplicación del presente Protocolo. La UNESCO prestará esa asistencia dentro de los límites de su programa y sus posibilidades.

2. Se insta a las Partes a proporcionar asistencia técnica bilateral o multilateral.

3. La UNESCO está autorizada a presentar, por propia iniciativa, propuestas sobre estas cuestiones a las Partes».

Sobre esta base jurídica, la UNESCO desarrolló una primera iniciativa en relación a Kosovo, elaborando en 2001 un escueto folleto informativo -en inglés, serbio y albanés- dirigido al personal internacional allí desplegado ${ }^{49}$. Pero será en Mali, sobre la base de la incorporación al mandato de la MINSUMA la protección «de los lugares de importancia cultural e histórica de Mali, en colaboración con la UNESCO», como hemos visto, donde la Organización desarrollará una labor más intensa en esta materia.

Así, en primer lugar, cabe mencionar que, en noviembre de 2012, el Ministerio de Cultura de Mali con el apoyo de la UNESCO elaboró un muy valioso Passeport pour le patrimoine et les biens culturels à préserver du Nord du Mali: Tombouctou, Gao, Kidal ${ }^{50}$. Meses después, la UNESCO elaboró un folleto, mucho más amplio que el de 2001, dirigido expresamente al personal de la MINUSMA -militar, policial y civil- y sobre cuya base se inició el programa de capacitación de dicho personal en octubre de 2013 y que continuará mientras la operación siga desplegada en Mali ${ }^{51}$.

49 Doc. CLT/CH/01/7.1/INF1, de 2001, <www.unesco.org/new/fileadmin/MULTIMEDIA/ HQ/CLT/pdf/leaflet-eng-albanais-serbe_2001.pdf>.

$50<$ www.unesco.org/new/fileadmin/MULTIMEDIA/HQ/BPI/EPA/images/media_services/Director-General/Passeport-pour-le-Mali.PDF>.

51 Como resume el Secretario General de Naciones Unidas: «A través de la labor de la Organización de las Naciones Unidas para la Educación, la Ciencia y la Cultura, las Naciones Unidas han elaborado módulos de capacitación para el personal militar, policial y civil sobre sus responsabilidades y deberes en lo que respecta a la protección del patrimonio cultural de Malí». Informe del Secretario General sobre la situación en Malí, S/2013/582, de 1 de octubre, par. 51. 
Ambos folletos, en unas pocas páginas, muy gráficas -con gran cantidad de fotos y mapas-, fáciles de entender y retener, muestran el patrimonio material e inmaterial de Mali, así como las obligaciones básicas contenidas en la normativa internacional para el respeto, salvaguardia y protección del patrimonio cultural del país donde la operación está desplegada. Obligaciones reforzadas con la incorporación del deber de cuidado en las resoluciones del Consejo de Seguridad relativas a la Operación, como hemos visto. En su informe de diciembre de 2015, el Secretario General de las Naciones Unidas resumía así los logros del Programa: «La MINUSMA siguió examinando el impacto ambiental de las operaciones en las proximidades de sitios culturales e históricos. Entre el 23 de septiembre y el 7 de diciembre, la Misión capacitó a 756 miembros de su personal en cuestiones relativas a la gestión ambiental y cultural, con arreglo a las disposiciones de la política ambiental del Departamento de Apoyo a las Actividades sobre el Terreno y el Departamento de Operaciones de Mantenimiento de la Paz para las misiones de las Naciones Unidas sobre el terreno» ${ }^{52}$.

$\mathrm{Y}$ es que, los resultados de la cooperación entre la UNESCO y la MINUSMA han sido tan positivos que en su Estrategia para reforzar la labor de la UNESCO en materia de protección de la cultura y promoción del pluralismo cultural en caso de conflicto armado la UNESCO anuncia: «Se intensificará la cooperación con las fuerzas armadas, incluidas las fuerzas de mantenimiento de la paz de las $\mathrm{Na}$ ciones Unidas, con el fin de aumentar el conocimiento y el entendimiento del derecho bumanitario en relación con la protección del patrimonio cultural durante los conflictos. La UNESCO aprovechará la experiencia positiva que supuso la aplicación de la resolución 2100 (2013) del Consejo de Seguridad de las Naciones Unidas, en virtud de la cual se estableció la Misión Multidimensional Integrada de Estabilización de las Naciones Unidas en Malí (MINUSMA)» ${ }^{53}$.

\subsection{La acción de la UNESCO para la reconstrucción del patrimonio cultural de Mali destruido o dañado en el conflicto}

Junto a la labor de sensibilización y capacitación del personal de la MINUSMA, ante la crisis de Mali la UNESCO ha desarrollado una muy importante labor, primero de denuncia y posteriormente de reconstrucción. Así, y

52 Informe del Secretario General sobre la situación en Malí, S/2015/1030, de 24 de diciembre, par. 48.

53 «Refuerzo de la labor de la UNESCO en materia de protección de la cultura...», op. cit., par. 35. Cursivas añadidas. 
por lo que a la denuncia respecta, ya el 3 de abril de 2012 su Directora General, Irina BOKOVA, realizó un primer llamamiento para la preservación del Patrimonio Mundial de Tombuctú ${ }^{54}$ y el 15 de abril llamó a una acción concertada para evitar la pérdida de su patrimonio ${ }^{55}$; tal y como prevé el artículo 31 del Protocolo 2 del Convenio de La Haya de $1954^{56}$, y tal y como posibilitaba la incorporación, desde su mismo establecimiento, de la función de protección del patrimonio cultural de Mali en el mandato de la MINUSMA.

Acción concertada que acabaría llegando, una vez restaurado el control y la seguridad sobre la zona, a partir del 19 de febrero de 2013, cuando se adoptó el Plan de Acción para el patrimonio cultural de Mali57. Así, como explicaba el Secretario General en su informe de octubre de 2013: «Las Naciones Unidas han seguido evaluando los sitios históricos y del patrimonio cultural en Gao y Tombuctú con miras a su rehabilitación. Se han definido medidas prioritarias para salvaguardar los manuscritos antiguos de Tombuctú. Los trabajos en curso incluyen el enlucido anual de la mezquita de Djingareyber en Tombuctú y un estudio arquitectónico de todos los mausoleos destruidos. (...). El Servicio de Actividades Relativas a las Minas también ha colaborado con los asociados de las Naciones Unidas para proteger los sitios del Patrimonio Mundial en Malí» ${ }^{58}$.

En los primeros meses de 2014 se pusieron en marcha los diferentes programas de reconstrucción y recuperación del patrimonio cultural destruido o perdido: «El 11 de febrero, el Ministerio de Cultura y la Organización de las Naciones Unidas para la Educación, la Ciencia y la Cultura (UNESCO) llevaron a cabo una misión de evaluación conjunta en Gao. En opinión de los expertos, deberían realizarse importantes trabajos de conservación en la Tumba de los Askia, declarada Patrimonio de la Humanidad, antes de que llegue la próxima estación de lluvias, para así evitar que siga deteriorándose. Estos

$54<$ www.unesco.org/new/es/media-services/single-view/news/director_general_of_unesco_urges_respect_for_the_preservation_of_world_heritage_site_of_timbuktu_mali/\#.V4Sz3 _ mLTcs $>$.

$55<$ www.unesco.org/new/en/media-services/single-view/news/unesco_director_general_appeals_ for_concerted_action_to_prevent_loss_or_destruction_of_timbuktus_documentary_heritage/\#.V4S2zvmLTcs>.

56 «En casos de graves violaciones del presente Protocolo, las Partes se comprometen a actuar conjuntamente por conducto del Comité o por separado, en colaboración con la UNESCO y las Naciones Unidas y de conformidad con la Carta de las Naciones Unidas».

$57<$ whc.unesco.org/en/news/987/>.

58 Informe del Secretario General sobre la situación en Malí, S/2013/582, de 1 de octubre, par. 51. 
trabajos, que comenzarán en mayo, serán dirigidos por la comunidad local y especialistas en conservación de construcciones de barro. El 14 de marzo, durante una ceremonia celebrada en Tombuctú para marcar el inicio oficial de las labores de reconstrucción del patrimonio maliense, la UNESCO presentó su estrategia para la reconstrucción de los mausoleos, en que se describen las actividades que se desarrollarán en los próximos cuatro años para rehabilitar completamente el patrimonio nacional que se encuentra en mal estado. En enero, la MINUSMA prestó apoyo logístico para que el personal del Centro de Documentación e Investigación Ahmed Baba encargado de investigar y conservar los manuscritos antiguos de Malí pudiera regresar a Tombuctú $\gg^{59}$.

Desarrollos conjuntos, como vemos, entre la UNESCO, la MINUSMA, las autoridades locales y la propia población de Mali para la localización y recuperación de manuscritos y bibliotecas antiguas ${ }^{60}$, reconstrucción de mausoleos y mezquitas en Gao y Tombuctú, y protección frente al saqueo de sitios arqueológicos de las regiones de Kidal y Gao, que muestran un trabajo intenso, coordinado y de gran eficacia, que ha permitido recuperar muy rápidamente buena parte del patrimonio dañado, perdido o destruido para los pueblos que conforman Mali. Baste los siguientes relatos en respectivos informes del Secretario General dando cuenta de los avances para ejemplificarlo:

«El 13 de octubre (de 2014) la MINUSMA y la Organización de las Naciones Unidas para la Educación, la Ciencia y la Cultura (UNESCO) dieron comienzo a un proyecto de efecto rápido para rehabilitar cuatro bibliotecas privadas en Tombuktú, donde se guardan manuscritos antiguos. El 3 de julio (de 2014), en Gao, la UNESCO, con apoyo logístico de la MINUSMA, emprendió los trabajos de nuevo enlucido de la tumba de los Askia, que figura en la lista del patrimonio mundial» ${ }^{61}$.

«El 8 de abril (de 2015), la MINUSMA visitó el emplazamiento arqueológico de EsSouk (región de Kidal) y confirmó las denuncias de saqueo

59 Informe del Secretario General sobre la situación en Malí, S/2014/229, de 28 de marzo, par. 47.

60 Por cierto, de especial relevancia histórica para España, puesto que, como explica BoLAÑos MARTÍNEZ: «Paradojas de la historia, una parte importante del legado a merced de los salafistas, tiene su origen en la persecución del Islam en la Península Ibérica. No hay que olvidar que en Tombuctú se conserva una importante clave para el estudio del exilio morisco medieval español: el Fondo Kati, una impresionante biblioteca de más de 3.000 manuscritos en árabe, español, hebreo y francés perteneciente a una familia maliense de orígenes andalusíes». BolaÑos MARTíNEZ, J., «Integrismo y patrimonio cultural», Revista Española de Defensa (octubre de 2012), p. 51.

61 Informe del Secretario General sobre la situación en Malí, S/2014/943, de 23 de diciembre, par. 42. 
realizadas por la población local. EsSouk figura en la lista tentativa de los emplazamientos de Malí para su inclusión en la Lista del Patrimonio de la Humanidad de la UNESCO $\gg^{62}$.

«Los días 15 de junio y 13 de agosto (de 2015), miembros de la MINUSMA visitaron Gao en coordinación con el Ministerio de Cultura, Artesanía y Turismo para demarcar el sitio arqueológico de Saneye, situado a $6 \mathrm{~km}$ de la ciudad, a fin de protegerlo del saqueo, dado que ya había sufrido saqueos y excavaciones ilícitas» ${ }^{63}$.

Esfuerzo concertado que ha permitido, en fin, que el 7 de septiembre de 2015 -apenas 3 años después de su destrucción- estuviesen reconstruidos todos los mausoleos destruidos en Tombuctú, mientras que la reconstrucción de las mezquitas dañadas continúa y se espera completar en breve. Como acaba de señalar el Comité del Patrimonio Mundial, en su reunión de julio de 2016 en Estambul:

«The results achieved by the project are greatly salutary, as all the 14 destroyed mausoleums were completely reconstructed. It was a true architectural challenge, with some of the mausoleums dating back to the 13th century. Over and above the fact that the reconstruction work has enabled the mausoleums to recover their authenticity of use and functionality, and restore the integrity of a major component of the property, it contributes to restoring social cohesion and peace within the communities of Timbuktu» ${ }^{64}$.

\section{La aCtuación de la Corte Penal Internacional: LA LUCHA CONTRA LA IMPUNIDAD EN MATERIA DE PROTECCIÓN DEL PATRIONIO CULTURAL}

La salvaguardia del patrimonio cultural, la restauración de la cohesión social y de la paz no sólo se consigue con la recuperación y protección del patrimonio dañado o destruido. La lucha contra la impunidad de los actos de destrucción deliberada del patrimonio de un pueblo a través del establecimien-

62 Informe del Secretario General sobre la situación en Malí, S/2015/426, de 11 de junio, par. 46.

63 Informe del Secretario General sobre la situación en Malí, S/2015/732, de 22 de septiembre, par. 51.

64 World Heritage Committee, Fortieth session Istanbul, Turkey 10-20 July 2016, doc. WHC/16/40.COM/7A.Add, 10 de junio de 2016, p. 7 (cursivas añadidas). 
to de responsabilidad penal individual es también esencial. Y es que, la destrucción deliberada en conflictos armados de bienes culturales protegidos supone la comisión de crímenes de guerra que deben ser perseguidos y castigados por los Estados o, complementariamente, por la Corte Penal Internacional, tal y como se tipifica en los artículos 8.2.b.ix y 8.2.e.iv del Estatuto de Roma.

Este último es precisamente el caso de Mali, lo que permite mostrar la tercera de las vías a través de la cual la Comunidad Internacional actúa en materia de protección del patrimonio cultural; en este caso, a través de la lucha contra la impunidad en este ámbito. Y lo hace, además y de nuevo, de manera concertada: a través de la Corte Penal Internacional, con la colaboración de la MINUSMA -en cuyo mandato, como hemos visto, se incorpora dicha función por primera vez en una operación de paz de Naciones Unidas- y de la UNESCO; con efectos que, de nuevo, van más allá del caso concreto, al mostrar el camino a seguir en el futuro.

Así, como explica la propia Organización: «La UNESCO ha comenzado también a cooperar más con la Corte Penal Internacional en lo que respecta a las investigaciones de esta, de conformidad con el artículo 8.2.e) iv) de su Estatuto, en que se consideran crímenes de guerra los ataques directos contra edificios dedicados a la religión, la educación, las artes, las ciencias o la beneficencia y los monumentos históricos. La cooperación conjunta entre la UNESCO y la Corte Penal Internacional en el caso de Malí, que se inició en 2012 tras la destrucción deliberada del patrimonio cultural de Tombuctú, consistió en proporcionar a la Corte Penal Internacional documentación detallada sobre el patrimonio cultural. Además, esta cooperación ha permitido crear una base sólida para seguir colaborando, en especial en los casos de países que no han ratificado aún las convenciones pertinentes o que no son partes en la Corte Penal Internacional $\gg^{65}$.

\subsection{La situación de Mali ante la Corte Penal Internacional}

Mali fue uno de los primeros Estados en incorporarse al Estatuto de la Corte Penal Internacional (CPI), firmándolo el 17 de julio de 1998 -el mismo día de su adopción en Roma- y ratificándolo el 16 de agosto de $2000^{66}$. Por

65 «Refuerzo de la labor de la UNESCO en materia de protección de la cultura...», op. cit., par. 29.

$66<$ https://treaties.un.org/Pages/ViewDetails.aspx?src=TREATY\&mtdsg_no=XVIII$10 \&$ chapter $=18 \&$ lang $=$ en $>$. 
consiguiente, y desde su misma entrada en vigor, el 1 de julio de 2002, la CPI tiene jurisdicción sobre los crímenes de su competencia cometidos en territorio de Mali.

El 18 de julio de 2012, a consecuencia de los crímenes que se estaban cometiendo en el contexto del conflicto armado interno iniciado meses antes, el propio Gobierno de Mali refirió a la CPI la investigación y enjuiciamiento de los crímenes cometidos desde enero de ese año, debido a la imposibilidad por parte de las autoridades judiciales de Mali de hacerlo. En dicho documento, el Ministro de Justicia hacía referencia, entre otros, a la destrucción de iglesias, mausoleos y mezquitas ${ }^{67}$. De hecho, apenas una semana antes, el 5 de julio, el Consejo de Seguridad se había hecho eco de la destrucción de lugares santos y de importancia histórica y cultural, advirtiendo que tales actos podían constituir violaciones del Derecho Internacional Humanitario:

«Condenando enérgicamente la profanación, el daño y la destrucción de lugares santos y lugares de importancia histórica y cultural, en especial, pero no exclusivamente, los declarados Patrimonio Mundial por la UNESCO, incluidos los de la ciudad de Tombuctú,

(...)

Habiendo determinado que la situación en Malí constituye una amenaza para la paz y la seguridad internacionales en la región,

Actuando en virtud del capítulo VII de la Carta de las Naciones Unidas,

16. Destaca que los ataques contra edificios dedicados a la religión o contra monumentos históricos pueden constituir violaciones del derecho internacional contempladas en el Protocolo Adicional II de los Convenios de Ginebra de 1949 y el Estatuto de Roma de la Corte Penal Internacional, en que Malí es Estado Parte, e insta además a todas las partes de Malí a que adopten de inmediato medidas apropiadas para asegurar la protección de los lugares de Malí declarados Patrimonio Mundial» ${ }^{68}$.

En consecuencia, la Fiscalía de la Corte abrió, con base en los artículos 14 y 15 del Estatuto de Roma, el correspondiente Examen Preliminar sobre la Situación de Mali sobre los hechos acaecidos a partir de enero de 2012 que pu-

67 Carta de remisión a la CPI del Ministro de fusticia de Mali, de 13 de julio de 2012, <www.icc-cpi.int/ NR/rdonlyres/A245A47F-BFD1-45B6-891C-3BCB5B173F57/0/ReferralLetterMali130712. pdf>.

68 S/RES/2056 (2012), de 5 de julio. 
dieran constituir crímenes para cuyo enjuiciamiento la CPI es competente. Examen Preliminar que culminó con el preceptivo Informe del artículo 53(1), en el que después de estudiar, en primer lugar, las precondiciones de jurisdicción y la competencia material de la Corte; en segundo lugar, las condiciones de admisibilidad tanto desde el punto de vista del principio de complementariedad como de la gravedad; y, finalmente, el interés de la justicia, concluyó que se daban las condiciones para la apertura de un investigación en la Situación de Mali:

$\ll 173$. The information available provides a reasonable basis to believe that war crimes have been committed in the context of the Situation in Mali since $\mathrm{Fa}$ nuary 2012, namely: (1) murder constituting war crime under Article 8(2) (c)(i); (2) mutilation, cruel treatment and torture constituting war crimes under Article 8(2)(c)(i) (3); the passing of sentences and the carrying out of executions without due process constituting war crime under Article 8(2)(c) (iv); (4) intentionally directing attacks against protected objects constituting war crimes under Article 8(2)(e)(iv); (5) pillaging constituting war crime under Article 8(2)(e)(v); and (6) rape constituting war crimes under Article 8(2)(e) (vi). This assessment is not binding for the purpose of any future investigation or selection of charges.

174. Since no national proceedings are pending in Mali or any other State against individuals who appear to bear the greatest responsibility for the most serious crimes committed in Mali, the Office has determined that the potential cases that would likely arise from an investigation into the situation would be admissible. Such cases, moreover, appear to be grave enough to warrant further action by the Court.

175. Since there are no substantial reasons to believe that such would not be in the interests of justice, the Prosecutor has decided to open an investigation into the Situation in Mali since fanuary 2012» ${ }^{69}$.

De todo ello, conviene destacar la afirmación de la Fiscal, Fatou BENSOUDA, de considerar que hay base razonable para creer que entre los crímenes de guerra que se han cometido en Mali se encuentra: «Dirigir intencionalmente ataques contra edificios dedicados a la religión, la educación, las artes, las ciencias o la beneficencia, los monumentos históricos, los hospitales y otros lugares en que se agrupa a enfermos y heridos, a condición de que no sean objetivos militares» ${ }^{70}$.

\footnotetext{
69 The Office of the Prosecutor, Situation in Mali. Article 53(1) Report, 16 de enero de 2013, par. 173-175. Cursivas añadidas, <www.icc-cpi.int/itemsDocuments/SASMaliArticle53_1PublicReportENG16Jan2013.pdf>.

70 Artículo 8(2)(e)(iv) del Estatuto de Roma.
} 
3.2. El asunto Fiscalía v. Ahmad Al Faqi Al Mabdi: primer caso ante la Corte Penal Internacional de crímenes de guerra por la destrucción de bienes protegidos

Sobre esta base procesal, el 18 de septiembre de 2015 y a solicitud de la Fiscalía, la Sala de Cuestiones Preliminares I en la Situación de Mali emitió orden de arresto contra Ahmad Al Faqi Al Mahdi por haber cometido, individualmente o conjuntamente con otros, por haber ayudado o de otro modo contribuido a que se cometieran crímenes de guerra por los ataques dirigidos intencionalmente contra edificios consagrados a la religión y/o contra monumentos históricos ${ }^{71}$. Orden de arresto que fue comunicada al gobierno de Níger, país donde se encontraba detenido en ese momento $\mathrm{Al}$ Mahdi.

Níger entregó a Al Mahdi a la Corte apenas una semana después, el 26 de septiembre de 2015, y el 24 de marzo de 2016, ante las pruebas presentadas por la Fiscalía, la Sala de Cuestiones Preliminares I emitió la Decisión de confirmación de cargos contra Abmad Al Faqi Al Mabdi, de acuerdo con lo establecido en el artículo 61.7 del Estatuto de Roma. En ella se establece que entre abril de 2012 y enero de 2013 Tombuctú se encontraba bajo el control efectivo de AQMI y Ansar Dine, los cuales establecieron durante ese tiempo una estructura administrativa en la ciudad que incluía, entre otros, la denominada Hisbab o «Brigada de costumbres». Y que entre los meses de junio y julio de 2012 se llevó a cabo intencionalmente la destrucción total o daños severos de los siguientes edificios:

«(i) the Sidi Mahamoud Ben Omar Mohamed Aquit Mausoleum; (ii) the Sheikh Mohamed Mahmoud Al Arawani Mausoleum; (iii) the Sheikh Sidi El Mokhtar Ben Sidi Mouhammad Al Kabir Al Kounti Mausoleum; (iv) the Alpha Moya Mausoleum; (v) the Sheikh Mouhamad El Mikki Mausoleum; (vi) the Sheikh Abdoul Kassim Attouaty Mausoleum; (vii) the Sheikh Sidi Ahmed Ben Amar Arragadi Mausoleum; (viii) the door of the Sidi Yahia Mosque; (ix) the Bahaber Babadié Mausoleum and (x) the Ahmed Fulane Mausoleum, both adjoining the Djingareyber Mosque» ${ }^{72}$.

71 Chambre Préliminaire I, Situation en République du Mali, affaire le Procureur C. Ahmad Al Faqi Al Mahdi, Mandat d'arrêt à l'encontre d'Ahmad AL FAQI AL MAHDI, doc. ICC-01/1201/15, 18 de septiembre de 2015, p. 8, <www.icc-cpi.int/CourtRecords/CR2015_18211.PDF>.

72 Pre-Trial Chamber I, Situation in the Republic of Mali in the Case of the Prosecutor V. Ahmad Al Faqi Al Mahdi, Decision on the confirmation of charges against Abmad Al Faqi Al Mahdi, doc. ICC-01/12-01/15, 24 de marzo de 2016, par. 34, <www.icc-cpi.int/CourtRecords/ CR2016_02424.PDF>. 
Edificios que eran considerados y protegidos como una parte significativa del patrimonio cultural de Tombuctú y Mali y que, como hemos visto, habían sido clasificados como Patrimonio Mundial por la UNESCO. Destrucción que llevó a la UNESCO a incluir a Tombuctú dentro de la Lista de Patrimonio en Peligro el 28 de junio de $2012^{73}$. Junto a ello, la Sala de Cuestiones Preliminares I destaca para calificar los hechos como crimen de guerra de acuerdo con el artículo 8(2)(e)(iv) del Estatuto de Roma lo siguiente:

«39. The unanimous outcry of the international community and individuals concerned substantiates the Prosecutor's allegation as to the seriousness of the acts. The evidence submitted by the Prosecutor confirms that the Buildings/Structures played an important role in the life of the inhabitants of Timbuktu and that their destruction was considered as a serious matter and regarded by the local population as an aggression towards their faith. Some of the Buildings/Structures have since been reconstructed, while in other instances something symbolic was built.

(...)

41. It is not in dispute that the Buildings/Structures were dedicated to religion and constituted historic monuments because of their origins and significance, and that none of them constituted a military objective.

42. Further, the evidence is univocal in showing that the Buildings/Structures were specifically identified, chosen and targeted by the perpetrators as objects of their attack, precisely in light and because of their religious and historical

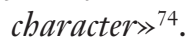

Pues bien, Ahmad Al Faqi Al Mahdi -maliense nacido a unos $100 \mathrm{~km}$. de Tombuctú, habitante de la ciudad y descrito, como eminente profesor y experto en asuntos religiosos, como la persona más prominente y de mayor competencia en la materia en Tombuctú $u^{75}$ - actuó en cooperación con los líderes de los dos grupos citados que ocupaban la ciudad y jugó un papel muy relevante en las instituciones administrativas que éstos establecieron: fue quien constituyó la Hisbah a principios de abril de 2012 y la dirigió hasta septiembre de ese año,

$73<$ www.unesco.org/new/fr/media-services/single-view/news/des_sites_du_patrimoine_mondial_du_mali_inscrits_sur_la_liste_du_patrimoine_en_peril/\#.V4PGQ_mLTcs>.

74 Pre-Trial Chamber I, Situation in the Republic of Mali in the Case of the Prosecutor V. Ahmad Al Faqi Al Mahdi, Decision on the confirmation of charges against Abmad Al Faqi Al Mabdi, doc. ICC-01/12-01/15, 24 de marzo de 2016, par. 39-42. Cursivas añadidas.

75 Vid. ibid, par. 45. 
convirtiéndose para ello en miembro de Ansar Dine. Como jefe de la Hisbah, y tras intentar convencer a la población de que cesase en realizar sus prácticas tradicionales en relación con los mausoleos por juzgarlas heréticas, jugó un papel decisivo en llevar a cabo la destrucción de los Mausoleos y Mezquitas indicadas ${ }^{76}$, en la que, además, participó personalmente:

«In light of evidence outlined above, the Chamber is satisfied that Ahmad Al Faqi Al Mahdi is individually criminally responsible for the crime charged by the Prosecutor. Ahmad Al Faqi Al Mahdi was directly and personally involved in all stages of the (partial) destruction of the Buildings/ Structures. He was part of the planning phase - as religious expert and prominent personality in the context of the occupation of Timbuktu as well as of the preparatory and implementation phase - as head of the Hisbah» ${ }^{77}$.

Responsabilidad criminal que la Sala deja abierta en lo que a la forma de participación respecta, de acuerdo con el artículo 25.3 del Estatuto de Roma: ya como coautor (a); como inductor o proponente (b); como colaborador, cómplice o encubridor (c); o que de cualquier otro modo haya contribuido a la comisión del crimen (d). Y es que, como sostiene la propia Sala,

«when the evidence is sufficient to sustain each of the alternative forms of responsibility for the same conduct presented by the Prosecutor, it is appropriate that the charges be confirmed with the various available alternatives, in order for the Trial Chamber to determine which, if any, is established to the applicable standard of proof at $\operatorname{trial}^{78}{ }_{\gg}{ }^{79}$.

Finalmente indicar que, y por primera vez en la práctica de la CPI, el 1 de marzo de $2016 \mathrm{Al}$ Mahdi, asistido por sus abogados, expresamente

76 En concreto, Ahmad Al Faqi Al Mahdi «publicly declared that the decision to destroy the Buildings/Structures was deliberately taken: 'nous agissons ainsi parce que nous voulons la démolition des dômes'. He stated that the destruction of the domes had been ordered by 'le Messager' and not prohibited by the relevant texts consulted by him». Ibid, par. 49.

$77 \mathrm{Ibid}$, par. 55 .

78 Como, por lo demás, es la práctica seguida por la Corte en otros asuntos: Ntaganda Confirmation Decision, para. 100; Gbagbo Confirmation Decision, para. 227; Bemba et al. Confirmation Decision; Blé Goudé Confirmation Decision, para. 133; Ongwen Confirmation Decision, para. 35.

79 Pre-Trial Chamber I, Situation in the Republic of Mali in the Case of the Prosecutor V. Ahmad Al Faqi Al Mahdi, Decision on the confirmation of charges against Abmad Al Faqi Al Mahdi, doc. ICC-01/12-01/15, 24 de marzo de 2016, par. 22. 
se declaró culpable ante los jueces de la Corte. Como ha afirmado Fatou BENSOUDA:

«This is the first time that a suspect has expressed his intention to plead guilty to criminal conduct for which he is being prosecuted by my Office; a serious crime for which we had gathered overwhelming evidence. Such an admission of guilt, provided for in article 65 of the Rome Statute, will be a milestone in the history of the ICC. This case is also unprecedented in terms of its expeditiousness and efficiency» ${ }^{80}$.

En efecto, el 1 de junio se fijó la fecha del juicio oral ${ }^{81}$, que se inició el 22 de agosto y finalizó dos días después, dejándolo visto para sentencia que se haría pública el 27 de septiembre de $2016^{82}$ : justo un año después de que se emitiera la orden de arresto contra $\mathrm{Al}$ Mahdi y apenas 4 años después de ocurridos los hechos por los que se le ha juzgado. En la Sentencia, el Tribunal condena a Al Mahdi a 9 años de cárcel, al considerar, de un lado, la gravedad de los crímenes y, de otro, algunas circunstancias atenuantes:

«The Chamber finds that the crime for which Mr Al Mahdi is being convicted is of significant gravity. This said, the Chamber has found no aggravating circumstances and five mitigating circumstances, namely: (i) $\mathrm{Mr}$ Al Mahdi's admission of guilt; (ii) his cooperation with the Prosecution; (iii) the remorse and the empathy he expressed for the victims; (iv) his initial reluctance to commit the crime and the steps he took to limit the damage caused; and (v), even if of limited importance, his good behaviour in detention despite his family situation. Taking into account all these factors, the Chamber sentences $\mathrm{Mr} \mathrm{Al}$ Mahdi to 9 years of imprisonment» ${ }^{83}$.

80 Statement of the Prosecutor of the International Criminal Court, Fatou Bensouda, following admission of guilt by the accused in Mali war crime case: «An important step for the victims, and another first for the ICC», 24 de marzo de 2016, <www.icc-cpi.int/Pages/item.aspx?name=160324-otp-stat-alMahdi>.

81 Trial Chamber VIII, Situation in the Republic of Mali in the Case of the Prosecutor V. Ahmad Al Faqi Al Mahdi, Decision Setting the Commencement Date of the Trial, doc. ICC-01/12-01/15-93, 1 de junio de 2016, <www.icc-cpi.int/CourtRecords/CR2016_03987.PDF>.

82 Doc. ICC-CPI-20160824-PR1237, 24 August 2016, <www.icc-cpi.int//Pages/item.aspx?name $=$ pr1237 $>$.

83 Fudgment and Sentence in the case of the Prosecutor v. Ahmad al Faqi al Mahdi, No.: ICC-01/1201/15 de 27 de septiembre de 2016, par. 109, <www.icc-cpi.int/CourtRecords/CR2016_07244. $\mathrm{PDF}>$. 


\section{CONSIDERACIONES FINALES}

Como sostiene KILA: «International cooperation is conditional for effective and sustainable results; this comprises the creation of civil, military and combined networks. (...). Undoubtedly, the best chance for implementing plans and methods for cultural heritage protection is through international interagency coordination, preferably between and across government institutions, including the military, and NGOs, including international organizations» ${ }^{84}$. En efecto, la resolución $38 \mathrm{C} / 48$, adoptada por la $38^{\mathrm{a}}$ Conferencia General de la UNESCO en noviembre de 2015, aprueba la Estrategia citada «en el entendimiento de que su aplicación se efectuará en total coordinación y colaboración con los Estados Miembros interesados y en el marco de los órganos de las Naciones Unidas y de su mandato ${ }^{85}$.

En todo ello, Mali aparece como paradigma: ejemplo de la acción decidida, rápida, multifacética y concertada de las distintas instituciones de la Comunidad Internacional, y en estrecha colaboración con las autoridades y expertos locales malienses, en materia de protección de bienes culturales en conflictos armados. Es verdad, por lo demás, que las circunstancias que rodean al conflicto en Mali, y Mali mismo -que por muchas e importantes razones no es Siria-, han permitido aquí el consenso político en la Comunidad Internacional, imprescindible para poder desarrollar tales actuaciones, que no se da en otros lugares.

Pero no es menos cierto que Mali marca un camino, es ejemplo y prueba de que, sobre la base del consenso político -siempre imprescindible desde el punto de vista jurídico y operacional, de la legalidad y de la eficacia-, la Comunidad Internacional tiene instrumentos suficientes para hacer frente a los peligros que en conflictos armados enfrenta el patrimonio de los Estados. Es prueba, además, de que la actuación a favor del patrimonio cultural no sólo tiene como objetivo la preservación y, en su caso, recuperación de los bienes culturales de un pueblo -y que, en ocasiones son, incluso, patrimonio mundial, como es el caso-; sino que, además, y de manera principal, forma parte del esfuerzo de la Comunidad Internacional para la pervivencia de los pueblos mismos y del Estado a través de la preservación de su cultura, que es su identi-

84 KILA, J. D., «Heritage under siege...», op. cit., pp. 198-199.

85 Resolución UNESCO, Refuerzo de la labor de la UNESCO en materia de protección de la cultura y promoción del pluralismo cultural en caso de conflicto armado, doc. $38 \mathrm{C} / 48$, de 17 de noviembre de 2015 , pto. 1 . 
dad, y, con ello, para la construcción de la paz: de una paz que, mucho más allá de la mera ausencia de violencia -paz en sentido negativo- sólo puede ser si es sostenible y en sentido positivo ${ }^{86}$. Para lo cual, la preservación de la identidad y memoria cultural de los pueblos que conforman el Estado, y del Estado mismo, es esencial ${ }^{87}$. Junto a ello, la acción contra el saqueo y el tráfico de bienes culturales resulta también esencial para reducir/evitar la financiación de grupos armados ilegales y, con ello, favorecer los dos fines anteriores: la paz y la preservación de la identidad cultural de los pueblos.

Como se indica en la tantas veces citada Estrategia de la UNESCO de 2015: «La magnitud de los ataques contra la cultura que presenciamos hoy en día y su carácter sistemático son un reflejo del fuerte vínculo que existe entre las dimensiones cultural, humanitaria y de seguridad de los conflictos. La protección del patrimonio y la diversidad culturales durante los conflictos se presenta en la actualidad como un aspecto elemental, no solo para mitigar la vulnerabilidad, sino también para romper un círculo de violencia en que los ataques contra la cultura contribuyen a fomentar aún más el odio, el sectarismo y la fragmentación en la sociedad, alimentando la inestabilidad y el conflicto permanentes. (...) la diversidad cultural y promover el pluralismo cultural mediante la salvaguarda del patrimonio material e inmaterial de las comunidades y la protección de los derechos humanos y las libertades fundamentales no es solo una emergencia cultural. Es una seguridad y un imperativo bumanitario en situaciones de conflicto y de transición, y un elemento esencial para garantizar la paz y el desarrollo sostenibles» ${ }^{88}$. Para concluir: «Dado que la destrucción del patrimonio cultural se encuentra en primera línea de los conflictos, también la protección del patrimonio cultural y la promoción del pluralismo cultural deben estar en primera línea de la consolidación de la paz» ${ }^{89}$.

Y para todo ello, la lucha contra la impunidad a través del establecimiento de responsabilidad penal para aquellos que hayan cometido crímenes de

86 Vid. VACAs FernándeZ, F., «Aproximación a los conceptos de paz y seguridad en el espacio iberoamericano», en C. Díaz Barrado y F. Vacas Fernández (dir.), Guía del espacio iberoamericano de paz, seguridad y defensa, Instituto Universitario General Gutiérrez Mellado de investigación sobre Paz, Seguridad y Defensa, Madrid, 2010, pp. 15-36.

87 Como afirma RUSH: «Preservation of cultural property can be critical for social restoration in a devastated community». RusH, L. W., «Cultural Property Protection as a Force Multiplier in Stability Operations», Military Review (March-April 2012), p. 42.

88 «Refuerzo de la labor de la UNESCO en materia de protección de la cultura...», op. cit., parrs. 8 y 10. Cursivas añadidas.

89 Ibid., par. 31. Cursivas añadidas. 
guerra a través de la destrucción, saqueo y/o tráfico ilícito de bienes culturales, resulta fundamental. De ahí la importancia de la actuación de la CPI, que en el asunto Al Mabdi establece un primer precedente que marca el camino en esta materia. Como afirmaba la Fiscal de la Corte, Fatou Bensouda, en la vista para la confirmación de los cargos a Ahmad Al Faqi Al Mahdi:

«Let us be clear: what is at stake here is not just walls and stones. The destroyed mausoleums were important, from a religious point of view, from an historical point of view, and from an identity point of view. Such an attack against buildings dedicated to religion and historic monuments falls into the category of crimes that destroy the roots of an entire people and profoundly and irremediably affect its social practices and structures. This is precisely why such acts constitute a crime under article 8(2)(e)(iv) of the Rome Statute.

Let there be no mistake: the charges we have brought against Mr Ahmad Al-Faqi Al Mahdi involve most serious crimes; they are about the destruction of irreplaceable historic monuments, and they are about a callous assault on the dignity and identity of entire populations, and their religious and historical roots. The inhabitants of Northern Mali, the main victims of these attacks, deserve to see justice done.

No longer should such reprehensible conduct go unpunished» ${ }^{90}$.

90 Statement of the Prosecutor of the International Criminal Court, Fatou Bensouda, at the opening of the confirmation of charges hearing in the case against Mr Abmad Al-Faqi Al Mahdi, 1 de marzo de 2016, $<$ www.icc-cpi.int/Pages/item.aspx? name=otp-stat-01-03-16>.

Vid. tambien Statement of the Prosecutor of the International Criminal Court, Fatou Bensouda, at the opening of Trial in the case against Mr Ahmad Al-Faqi Al Mabdi, 22 August 2016. 
